\title{
Influence des facteurs environnementaux sur le cycle sexuel de l'aulacodine (Thryonomys swinderianus, Temminck 1827) élevée en captivité étroite
}

\author{
A.J.L. OKON ${ }^{1 *}$, D. SORO ${ }^{1}$ et A. FANTODJI ${ }^{1}$ \\ ${ }^{1}$ Université d'Abobo-Adjamé, UFR SN-Laboratoire de Biologie et Cytologie Animale, 02 BP 801 Abidjan 02, \\ Côte d'Ivoire. \\ *Auteur correspondant ; E-mail : jlokon@yahoo.fr/abou.okon@etudiants.edu.ci,Cell :+22558863407
}

\section{RESUME}

L'étude de l'influence des facteurs environnementaux sur le comportement sexuel des aulacodines et l'évolution du cycle sexuel réalisés sur 190 femelles reparties dans les zones centre et sud de la Côte d'Ivoire, n'a pas révélé des effets significatifs marqués de façon spécifique. Cependant, nous avons constaté chez l'aulacodine, quelles que soient les saisons, un état d'agitation quasi permanent. Les variations des indices vulvaires ne sont pas régulières. Les femelles sont à $70 \%$ bloquées au stade de pro œstrus. Généralement, il est rare en accouplement permanent d'observer les femelles à l'état d'œstrus car la copulation à lieu prématurément au pro œstrus avec des conséquences parfois dommageables pour les femelles. Les anoestrus sont très présents. Ils sont observés à des taux d'humidité élevé $(69,05 \%$ - 80,48\%) et des températures comprises entre 27 et $28,62 \pm 0,8{ }^{\circ} \mathrm{C}$. L'activité sexuelle fut quant à elle observée pour des températures comprises entre 24,34 et $29^{\circ} \mathrm{C}$ et une humidité de l'air variant entre 54,97 à $85,16 \%$.

(C) 2014 International Formulae Group. All rights reserved.

Mots clés : Aulacodine, cycle sexuel, saison.

\section{INTRODUCTION}

Aujourd'hui, en Afrique de l'Ouest et Centrale, l'élevage de l'aulacode est une activité en pleine expansion grâce à l'amélioration des paramètres nutritionnels, reproductifs et sanitaires de l'espèce en captivité étroite (Adu et al., 2003; Annor et al., 2012 ; Poku et al., 2013 ; Karamoko et al., 2013 ; Karamoko et al., 2014 ; Soro et al., 2014). Cependant, les bonnes perspectives économiques que représente l'activité aulacodicole semblent être freinées par de nombreux facteurs. Il a été constaté que l'aulacode est physiologiquement caractérisé par de longues périodes d'infertilité qui pénalise la rentabilité des élevages et constituent donc l'un des freins majeurs au développement de l'aulacodiculture (Fantodji et al., 2004). Du fait de la fugacité et de l'imprécision des signes de chaleur chez l'aulacodine (femelle adulte), aucune méthode de détection des chaleurs n'a été mise au point pour les éleveurs. Ils ne disposent d'aucun repère permettant de définir un point de départ du cycle sexuel. L'infertilité et l'infécondité sont deux exemples d'entités pathologiques, qualifiées de «maladies de production » se caractérisant par leurs manifestations subcliniques et leurs origines multifactorielles.

Dans le souci de contribuer à relever le niveau de productivité de cette espèce animale, les modifications de l'aspect de la 
vulve et les changements observés dans le comportement sexuel des aulacodines ont été étudié sous l'influence de paramètres environnementaux. Ainsi, nous avons étudié l'impact des saisons, l'effet des changements de température et de l'humidité de l'air sur le comportement sexuel et les variations de l'indice vulvaire au cours du cycle sexuel des aulacodines.

\section{MATERIEL ET METHODES Zone d'étude}

L'étude s'est déroulée de janvier 2008 à janvier 2009 concomitamment dans les aulacoderies de l'Université Nangui Abrogoua (UNA), Ex-Université d'Abobo-adjamé située dans la région des lagunes, plus précisément à Abidjan et celle de la ferme de kami située à Yamoussoukro en région des lacs en zone soudano-guinéenne. Le climat en région des lagunes est du type subéquatorial, avec deux saisons humides (la grande saison mai-juillet et la petite saison octobre-novembre) et deux saisons sèches (la grande saison de décembreavril et la petite saison août-septembre). La précipitation moyenne annuelle est de 2100 $\mathrm{mm}$. La température moyenne annuelle est de 26,2 ${ }^{\circ} \mathrm{C}$. L'humidité relative ne descend jamais en dessous des $65 \%$. En région des lacs, on distingue quatre saisons: une grande saison sèche (novembre à février), une grande saison des pluies (mars à juin), une petite saison sèche (juillet à août) et une petite saison des pluies (septembre à octobre). Les précipitations varient entre 900 et $1100 \mathrm{~mm}$, mais sont erratiques. La température moyenne de la région est d'environ $26^{\circ} \mathrm{C}$ et l'humidité de l'air varie entre 75 et $85 \%$ avec des chutes à $40 \%$ en période d'harmattan et se situe entre 80 et $85 \%$ en période pluvieuse.

\section{Matériel biologique}

Un cheptel expérimental de 190 aulacodines et 50 aulacodins a été considéré selon les répartitions suivantes :

- aulacoderie de l'UNA : 120 femelles d'âge compris entre 4 et 36 mois et 30 mâles adultes âgés de 7 à 36 mois;
- aulacoderie de kami : 70 femelles d'âge compris entre 5 et 36 mois et 20 mâles âgés de 7 à 36 mois.

La ration alimentaire est composée de fourrages et d'un complément alimentaire constitué de grains de maïs $(80 \%)$, de la poudre de la cendre de coquille d'escargot calcinée $(5 \%)$, de sel de cuisine $(5 \%)$ et de folioles sèches de Leucaena leucocephala (10\%) à raison de 100 à $150 \mathrm{~g}$ par animal.

\section{Infrastructures et équipement d'élevage}

L'aulacoderie est un bâtiment d'élevage de forme ordinaire contenant les enclos (aulacodère).

Les enclos sont compartimentés de forme rectangulaire, avec une surface de base de $2 \mathrm{~m}$ (L) x $1 \mathrm{~m}$ (l) et une hauteur de 0,50 m. Les 2 compartiments communiquent par une ouverture carrée de $20 \mathrm{~cm}$ de côté. Cette ouverture permet le libre passage des aulacodes d'un compartiment à l'autre et permet d'assurer une bonne conduite de l'élevage. Chaque compartiment est muni d'une portion métallique de $90 \mathrm{~cm}$ x $40 \mathrm{~cm}$ fait en barres de fer à béton. Les enclos sont disposés en batteries d'enclos étagées de 3 niveaux, des mangeoires et abreuvoirs sont utilisés pour servir le concentré alimentaire et l'eau aux aulacodes. Un petit matériel d'élevage est utilisé pour l'entretien des aulacodes et de l'aulacoderie.

\section{Matériel technique}

Un hygromètre et un thermomètre ont été utilisés, installés dans chaque aulacoderie pour mesurer l'humidité et la température.

\section{Méthodes}

Sélection des aulacodes et conduite d'élevage

Les aulacodes reproducteurs sont sélectionnés dès l'âge de 3 mois pour les femelles et 5 mois pour les mâles et élevés séparément jusqu'à l'âge de mise en accouplement qui est de 5 à 6 mois pour les femelles et 7 à 8 mois pour les mâles. Les critères de choix sont les suivants : bon état de santé ; poids vif corporel appréciable et variant entre 1 et $2 \mathrm{~kg}$; origine généalogique 
pour éviter la consanguinité. Le mode d'accouplement en groupe (ou lots) polygame composé chacun d'un aulacodin et de 4 aulacodines est pratiqué. Les femelles ayant un poids vif corporel égal ou supérieur à 1,5 kg sont accouplées avec un mâle dont le poids vif corporel est supérieur à celui des femelles d'au moins $0,5 \mathrm{~kg}$. Ils sont installés dans des batteries d'enclos étagés.

La conduite de l'élevage proprement dite nécessite :

- des activités quotidiennes : inspection du cheptel tôt le matin pendant une demi-heure à une heure suivie du nettoyage des aulacodères, première distribution des fourrages suivie 2 à 3 heures après de la distribution des aliments concentrés (sel de cuisine, grains de maïs et autres). Une deuxième distribution des fourrages est effectuée le soir à partir de 17 h. De l'eau potable est distribuée à volonté aux aulacodes.

- des activités hebdomadaires et mensuelles: lavage des mangeoires et abreuvoirs 2 à 3 fois par semaine, désinfection des aulacodères 1 fois par semaine, désinfection de l'aulacoderie 1 fois par mois, déparasitage des aulacodes 1 fois par mois.

- à chaque mise bas et sevrage : notification, pesée des aulacodeaux et des mères, sexage et marquage.

- à chaque mortalité : notification, pesée, brève description des observations, établissement d'une fiche d'expédition à la clinique vétérinaire et/ou au laboratoire vétérinaire ou, à défaut, incinération du cadavre et désinfection de l'aulacodère.

Méthode d'étude du comportement sexuel des aulacodines au cours du cycle

Des observations ont été quotidiennement réalisées et nous avons reporté sur une fiche d'élevage portant le nom de la ferme, le jour, le numéro d'enclos, le nom de la femelle, son âge, son poids, nombre de parturition, l'aspect de la vulve, la température de l'air ambiante, l'humidité de l'air et les évènements (mise bas, signes d'accouplements, test de gestation, mort).
Méthode d'étude des modifications des aspects de la vulve au cours du cycle

Les variations périodiques de l'aspect de la vulve ont été appréciées par un indice vulvaire. Pour ce faire, un système de codification numérique selon le modèle proposé par Adjanohoun (1988) et modifié pour la circonstance, a été utilisé pour exprimer les modifications observées au niveau de la vulve comme suite :

- indice vulvaire (IV0), la vulve est fermée par une membrane vaginale continue;

- indice vulvaire (IV1), la vulve est fermée par une croûte tout le long;

- indice vulvaire (IV2), la vulve est ouverte et les lèvres congestionnées;

- indice vulvaire (IV3), la vulve est fermée partiellement par une croûte hémorragique.

\section{Méthode de codification des paramètres étudiés}

Dans les stations 1 et 2, nous avons identifié les saisons sèches par le code saison (1) et les saisons de pluies par saison (2). Aussi, nous avons identifié le caractère agité des femelles par (Cs1) et le caractère docile par (Cs2).

\section{Analyse statistique}

Les résultats ont été exprimés sous forme de moyennes \pm écart types. La comparaison des moyennes a été faite à l'aide $\mathrm{du}$ test d'ANOVA à $\mathrm{P}<0,05$. L'analyse statistique en général a été faite à l'aide de la version 7.1 de statistica.

\section{RESULTATS}

Influence des saisons sur le cycle sexuel des aulacodines

Nous avons observé pendant une année les vulves de 190 femelles dans les stations d'Abidjan et de Yamoussoukro.

Influence des saisons sur les variations des indices vulvaires

\section{En général}

L'analyse de la variance révèle l'existence d'effets significatifs marqués à 
$(\mathrm{F}=15,95 \mathrm{p}=0,00066)(\mathrm{p}<0,05)$ des variations saisonnières sur les indices vulvaires.

\section{De façon spécifique}

\section{Station 1}

L'analyse de la variance ne révèle pas d'effets significatifs marqués à $\mathrm{F}=2,94 \mathrm{p}=0,08$ ( $p>0,05)$ de l'influence saisonnière sur les indices vulvaires dans la station 1. L'indice vulvaire moyen au cours des saisons est $1,13 \pm$ 1,11. Les variations moyennes des indices vulvaires au cours des saisons sont représentées dans la Figure 1. Les différences des épaisseurs des bandes rouge et bleu traduisent la variabilité des indices vulvaires observés dans la station 1. Les variations des indices vulvaires ont été plus importantes en saison 1 qu'en saison 2. On note l'absence de l'indice vulvaire IV2 au cours de la saison 2 . Le stade IV1 est beaucoup présent avec un taux variant entre $34 \%$ et $48 \%$ des observations réalisées. Le stade IV0 est le second avec un taux d'environ $40 \%$ des observations réalisées. Le stade IV3 est le troisième avec des proportions avoisinantes les $20 \%$. De faibles taux des indices vulvaires IV2 ont été obtenus environ $10 \%$ sur l'ensemble des deux saisons.

Station 2

L'analyse de la variance révèle l'existence d'effets significatifs marqués à ( $\mathrm{F}=$ 195,27 $\mathrm{p}=0) \quad(\mathrm{p}<0,05)$ de l'influence des saisons sur les variations des indices vulvaires dans la station 2. L'indice vulvaire moyen est de 1,27 $\pm 1,03$ au cours des saisons. La Figure 2 montre aussi une variation des indices vulvaires au cours des saisons. Le stade IV1 est le plus observé au cours des saisons avec des taux variant entre $43 \%$ et $60 \%$. Le stade IV3 suit avec des proportions variantes entre $20 \%$ et $50 \%$. Le stade IV0 est le troisième avec des taux variant entre $8 \%$ et $23 \%$. Le stade IV2 est très peu représenté avec un taux d'environ 1\% (Figure 2).

\section{Conclusion partielle}

En général, l'analyse de la variance de l'influence des saisons sur les variations des indices vulvaires présente des effets significatifs marqués à $\mathrm{p}<0,05$. Cependant, l'analyse station par station indique le contraire. Ces effets sont marqués dans la station $2(\mathrm{p}<0,05)$ et non dans la station 1 $(p>0,05)$. Le stade IV1 reste dominant au cours des saisons et selon les stations. Il est suivi du stade IV0 dont les taux avoisinent les $30 \%$. Le stade IV2 n'est presque pas observé dans l'ensemble des stations.

\section{L'influence des saisons sur le comportement sexuel (Cs) des femelles En général}

L'analyse de la variance révèle l'existence d'effets significatifs marqués à $(\mathrm{F}=6,65 \mathrm{p}=0,0099)(\mathrm{p}<0,05)$ des saisons de l'année sur le comportement sexuel des femelles. En moyenne, les femelles ont été plus agitées que dociles au cours des saisons dans les deux stations (Cs moyen=1,16 \pm $0,37)$.

\section{De façon spécifique Station 1}

Les analyses de la variance révèle l'existence d'effets significatifs marqués à $(\mathrm{F}=4,43 \mathrm{p}=0,037) \quad(\mathrm{p}<0,05) \quad$ des variations saisonnières sur le comportement sexuel des femelles dans la station 1. En moyenne les femelles n'ont pas été réceptives aux mâles car le Cs moyen est de 1, $17 \pm 0,38$ dans la station 1. Cependant, nous remarquons que le taux de docilité (cs2) des femelles est quasiment identique au cours des saisons 1 et 2 estimé à environ $40 \%$ des observations effectuées. Au cours de la saison 1, le taux d'agitation (Cs1) a atteint $60 \%$ des observations (Figure 3).

Station 2

L'analyse de la variance ne révèle pas l'existence d'effets significatifs marqués à $(F=0,13 p=0,7) \quad(p>0,05)$. Des variations des saisons sur le comportement sexuel des femelles dans la station 2. Ici aussi, on note une non réceptivité des femelles en moyenne car le Cs moyen est de 1,13 $\pm 0,36$. Les femelles ont été réceptives à moins de $20 \%$ au cours des deux saisons. L'agitation des femelles a été plus observée en saison 1 qu'en 
saison 2 avec de taux avoisinants les $70 \%$ (Figure 4).

\section{Conclusion partielle}

L'analyse de la variance indique des effets marqués significatifs marqués de l'influence des saisons sur le comportement sexuel des femelles dans la station 1 et non dans la station 2. Le comportement sexuel moyen, supérieur à 1 dans les deux stations au cours des différentes saisons indique une agitation quasi permanente chez les aulacodines même si parfois des docilités relatives sont observées.

Influence des variations des températures sur le cycle sexuel des aulacodines

Influence des températures sur les variations des indices vulvaires

En général

L'analyse de la variance révèle des effets significatifs marqués $(\mathrm{F}=144,12, \mathrm{p}=0)$ $(\mathrm{p}<0,05)$ des variations des températures ambiantes au cours des saisons.

\section{De facon spécifique}

Station 1

L'analyse de la variance présente des effets significatifs marqués $(\mathrm{F}=42,58, \mathrm{p}=0)$ $(p<0,05)$ des variations des températures sur les indices vulvaires. Les températures dans la station 1 ont varié entre $22{ }^{\circ} \mathrm{C}$ et $34{ }^{\circ} \mathrm{C}$. Aux stades IV0 et IV3, les températures moyennes enregistrées furent respectivement de $27,13 \pm 0,8^{\circ} \mathrm{C}$ et $27,5 \pm 1,2{ }^{\circ} \mathrm{C}$. Au stade IV1, les températures moyennes sont de 26,94 \pm $1,48{ }^{\circ} \mathrm{C}$. Elles sont de l'ordre de 24,34 $\pm 2,6$ ${ }^{\circ} \mathrm{C}$ au stade IV2.

Station 2

L'analyse de la variance présente des effets significatifs marqués à $(F=97,93 \mathrm{p}=0)$ $(\mathrm{p}<0,05)$ des variations des températures sur les indices vulvaires des aulacodines dans la station 2 .

Les températures ont varié entre 22 et $34{ }^{\circ} \mathrm{C}$ au cours de l'année. Nous avons enregistré au stade IV0 une moyenne de température de $28,62 \pm 2,53{ }^{\circ} \mathrm{C}$. Elle fut de $29,36 \pm 3,11^{\circ} \mathrm{C}$ au stade IV1, de 26,65 $\pm 1,94$ ${ }^{\circ} \mathrm{C}$ au stade IV 3 contre $32{ }^{\circ} \mathrm{C}$ au stade IV2.

\section{Conclusion partielle}

L'analyse de la variance révèle des effets significatifs marqués $\mathrm{p}<0,05$ des variations de températures sur les changements des aspects de la vulve (IV) dans les stations 1 et 2 .

Influence des températures sur le comportement sexuel (CS) des femelles En général

L'analyse de la variance présente des effets significatifs marqués $(\mathrm{F}=26,88, \mathrm{p}=0)$ $(\mathrm{p}<0,05)$ des températures sur le comportement sexuel des femelles.

\section{De facon spécifique}

Station 1

L'analyse de la variance ne révèle pas d'effets significatifs marqués à $(F=0,01 \mathrm{p}=0,9)$ ( $p>0,05)$ de l'influence de la température sur le comportement sexuel des aulacodines dans la station 1. En effet, le comportement sexuel des femelles n'aurait pas été influencé par les changements de température vu que les températures moyennes sont équivalentes $\left(\mathrm{Cs} 1=27,10 \pm 1,71{ }^{\circ} \mathrm{C}\right.$ et $\mathrm{Cs} 2=27,09 \pm 1,66$ $\left.{ }^{\circ} \mathrm{C}\right)$.

Station 2

L'analyse de la variance révèle l'existence d'effets significatifs marqués ( $\mathrm{F}=$ $31,44 \mathrm{p}=0) \quad(\mathrm{p}<0,05) \quad$ des variations des températures sur le comportement sexuel des femelles dans la station 2. Le tableau des statistiques descriptives montre que les femelles étaient plus agitées pour des températures moyennes de 28,74 $\pm 3,09{ }^{\circ} \mathrm{C}$.

\section{Conclusion partielle}

L'analyse de la variance de l'influence possible des changements annuels de température au cours des saisons sur le cycle sexuel des aulacodines a révélé des effets significatifs marqués en station 2.

Influence des variations de l'humidité de l'air sur le cycle sexuel des aulacodines Influence des variations de l'humidité de l'air sur les indices vulvaires En général

L'analyse de la variance montre des effets significatifs marqués $(\mathrm{F}=46,94 \mathrm{p}=0)$ 
$(\mathrm{p}<0.05)$ des variations de l'humidité relative de l'air sur les changements des aspects de la vulve.

\section{De facon spécifique}

Station 1

L'analyse de la variance montre des effets significatifs marqués à $\mathrm{F}=58,28 \mathrm{p}=0$ $(\mathrm{p}<0,05)$ des modifications des aspects de la vulve au cours du cycle sexuel dans la station 1. L'humidité moyenne varie d'un indice vulvaire à l'autre. Ainsi, nous avons enregistré au stade IV0 un taux d'humidité moyen de $80,4 \pm 16,23 \%$, au stade IV1 un taux de 81,39 $\pm 14,59 \%$, au stade IV2 un taux de 63,02 \pm $22,04 \%$ et un taux de $85,16 \pm 9,93 \%$ au stade IV3.

Station 2

L'analyse de la variance révèle des effets significatifs marqués à $\mathrm{F}=55,20 \mathrm{p}=0$ $(\mathrm{p}<0,05)$ des variations de l'humidité relative de l'air sur les changements des aspects de la vulve. Au stade IV0, l'humidité moyenne enregistrée est $69,05 \pm 10,87 \%$. Elle est de $58,56 \pm 21,07 \%$ au stade IV1. Au stade IV2 elle tombe à $27 \%$ tandis qu'au stade IV3, elle vaut $54,9 \pm 13,03 \%$.

\section{Conclusion partielle}

L'étude de l'influence éventuelle des variations de l'humidité de l'air au cours des saisons sur les indices vulvaires révèle des effets significatifs marqués à $p<0,05$. Nous remarquons que contrairement à la station 1 ; le taux d'humidité moyen est d'environ $60 \%$ un peu plus faible en station 2. Les taux d'humidité des stades IV2 sont les plus bas dans les deux stations.

\section{L'influence de l'humidité de l'air sur le comportement sexuel des aulacodines En général}

L'analyse de la variance montre des effets significatifs marqués ( $\mathrm{F}=6,91 \mathrm{p}=0,0085)$ $(p<0,05)$ des variations de l'humidité de l'air sur les comportements sexuels des femelles.

\section{De facon spécifique}

Station 1

L'analyse de la variance ne révèle pas des effets significatifs marqués ( $\mathrm{F}=2,99$ $\mathrm{p}=0,08) \quad(\mathrm{p}>0,05)$. L'humidité moyenne enregistrée dans la station 1 pour des comportements d'agitation est de 81,80 \pm $14,88 \%$. Cette valeur est sensiblement égale à l'humidité moyenne obtenue pour des comportements de docilité $(80,72 \pm 14,19 \%)$. Station 2

L'analyse de la variance ne révèle pas des effets significatifs marqués ( $\mathrm{F}=7,11$ $\mathrm{p}=0,07)(\mathrm{p}>0,05)$. Nous avons obtenu qu'en moyenne les femelles furent agitées pour un taux de $59,26 \pm 19,09 \%$ et dociles pour un taux d'humidité moyen de $62,81 \pm 13,47 \%$.

\section{Conclusion partielle}

L'analyse de la variance ne révèle pas des effets significatifs marqués $(p>0,05)$ des variations de l'humidité de l'air sur le comportement sexuel des femelles dans les stations 1 et 2 .

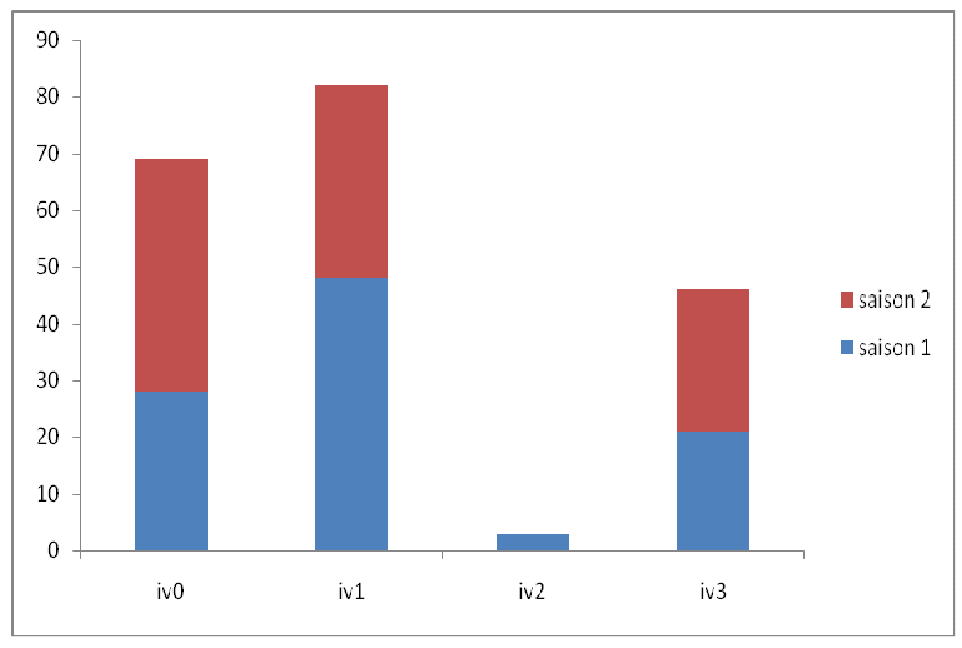

Figure 1: Variations des IV au cours des saisons dans la station 1. 
A.J.L. OKON et al. / Int. J. Biol. Chem. Sci. 8(2): 579-587, 2014

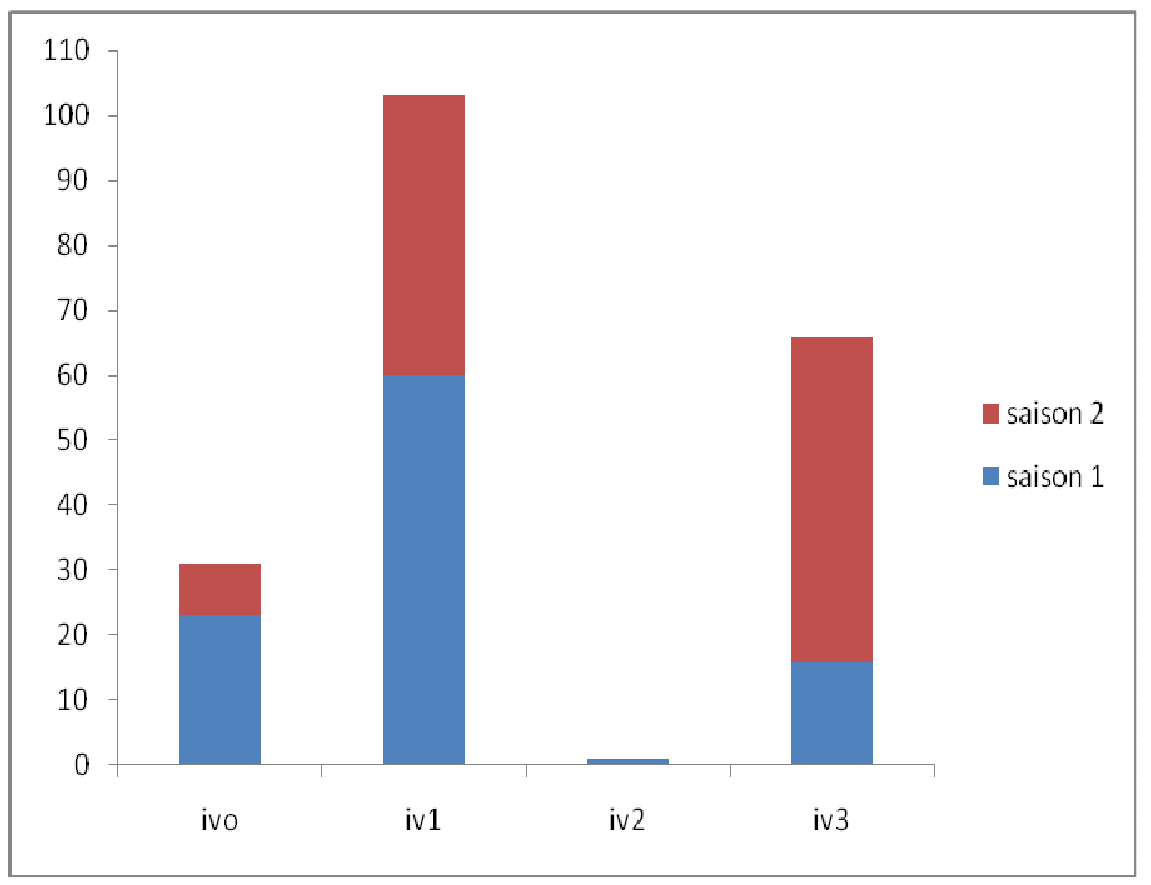

Figure 2: Variations des IV au cours des saisons dans la station 2.

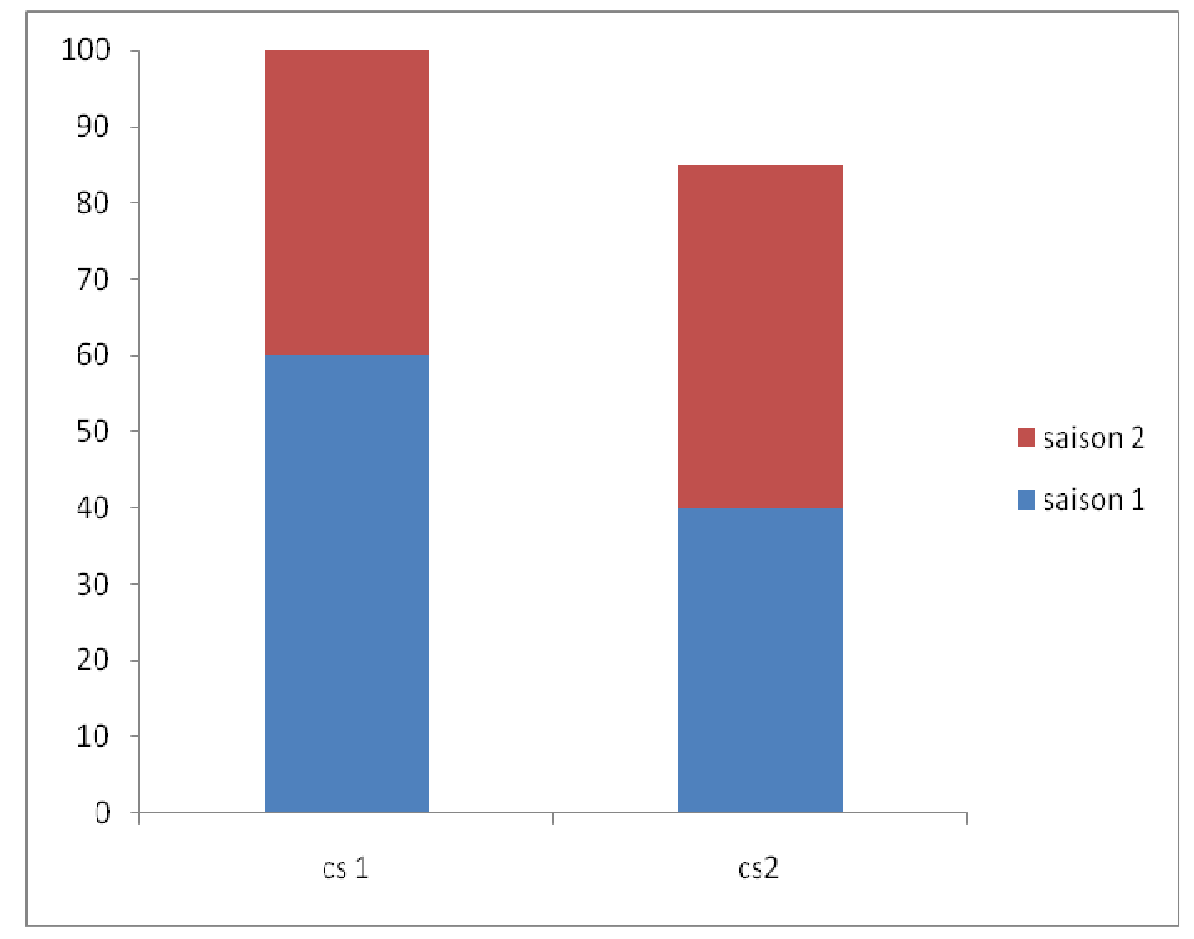

Figure 3: Variations des Cs au cours des saisons dans la station 1. 


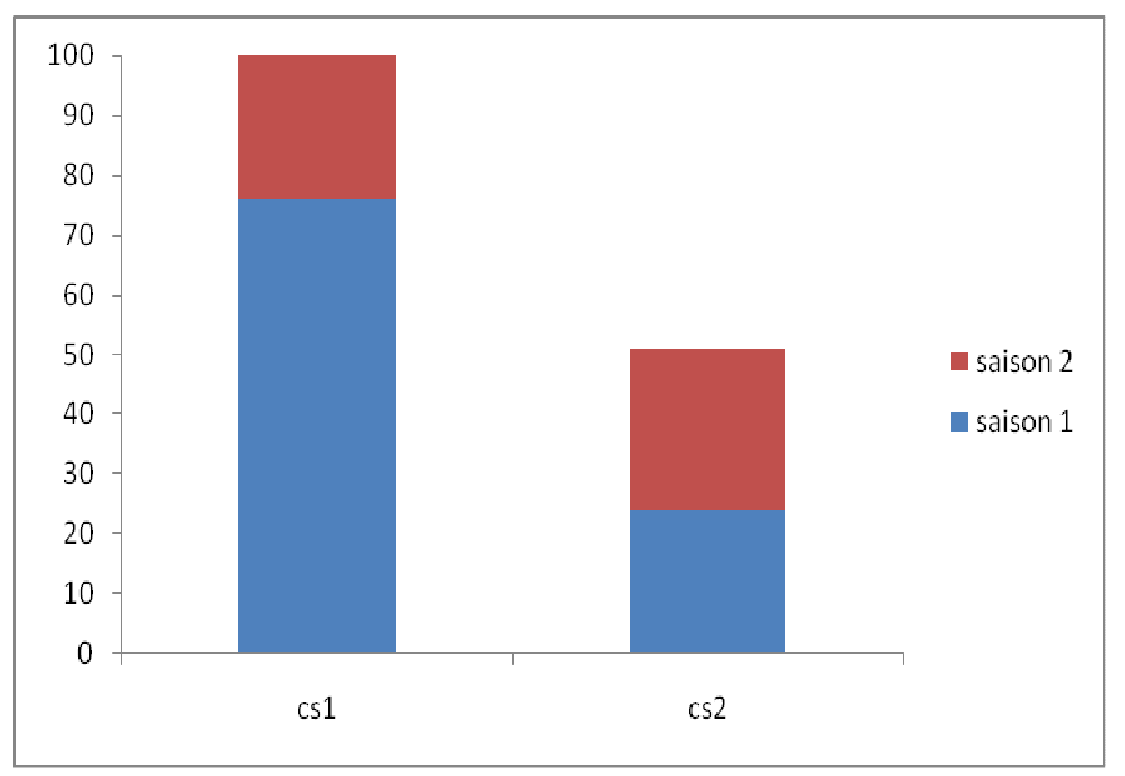

Figure 4: Variations des Cs au cours des saisons dans la station 2.

\section{DISCUSSION}

En général, les variations des saisons n'ont pas influencé le comportement sexuel et les indices vulvaires des aulacodines dans les deux stations comme l'a aussi constaté Adjanohoun (1988). Ce phénomène est commun aux espèces à ovulation provoquée que Gayrard (2007) a décrit chez la lapine et chez la chatte. Selon Lebas (2011), cela est dû au caractère atypique du cycle sexuel de la lapine qu'il caractérise d'espèce à œstrus et non à cycle sexuel. Cependant, Les variations des aspects de la vulve de l'aulacodine semblent quelque peu influencées par l'hygrométrie car les rares cas d'œstrus ont été observés pour des taux d'humidité les plus bas dans les deux stations. La différence est nette avec le stade d'œstrus qui intervient lorsqu'il fait moins chaud $\left(26,56{ }^{\circ} \mathrm{C}\right)$ et lorsque l'humidité de l'air est basse (54,97\%). L'expérience de Guerin et al. (1994) est à ce jour la seule étude selon Druet (2005) à tenter d'isoler le facteur température. Ainsi, au-delà d'une température minimale d'environ $9{ }^{\circ} \mathrm{C}$, on assiste chez la jument à une entrée en période ovulatoire. Amany (1973) a remarqué qu'à l'état sauvage, les variations saisonnières influençaient l'activité ovarienne des aulacodines comme chez les mammifères en général dont les cycles sexuels sont influencés par les facteurs environnementaux (photopériode, température, humidité, pluviométrie, alimentation) (Druet, 2005 ; Gayrard, 2007 ; Ali et al., 2011 ; Ghavi et al., 2013). Toutefois, il apparait évident dans plus de $70 \%$ des cas que les femelles restent longtemps bloquées au pro œstrus. Dans très peu de cas, elles évoluent vers l'œstrus qui est très rare car la copulation à lieu prématurément au pro œstrus. Nous serons même tentés de dire que la tendance à manifester l'œstrus dépend de certains facteurs liés au nombre de parturition, au poids et à l'âge de l'animal. Car les femelles qui ont manifesté l'œstrus étaient à plus de d'une mise bas et d'âge supérieur à 15 mois.

\section{Conclusion}

Nous pouvons conclure au terme de cette étude que les variations des indices vulvaires et les comportements sexuels des femelles au cours de la reproduction chez l'aulacode sont peu influencés par les variations saisonnières. Toutefois, l'œstrus au 
cours du cycle sexuel de l'aulacodine apparaît pour des valeurs relativement basses des températures et de l'humidité de l'air.

\section{REFERENCES}

Adjanohoun E. 1988. Contribution au développement de l'élevage de l'aulacode (Thryonomys swinderianus, Temminck, 1827) et à l'étude de sa reproduction. Thèse, Ecole Nationale Vétérinaire d'Alfort, France, p. 198.

Adu EK, Aniny KG, Wallace PA, Ocloo TO. 2003. Reproduction and mortality in a colony of captive greater cane rats (Thryonomys swinderianus, Temminck, 1827). Trop. Anim. Health Prod., 32(1): 11-17.

Ali MN, Onyeanusi BI, Ayo JO, Oja SA, Salami SO, Nzalak JO, Byanet O. 2011. Effect of season on the female African Giant Rat (Cricetomys gambianus) in Zaria, Nigeria. Int. J. Morphol., 29(3): 841-844.

Amany KJ. 1973. Etude des populations d'aulacodes dans les savanes de Lamto. Mémoire de DEA, Université d'Abidjan, p.98.

Annor SY, Ahunu BK, Aboagye GS, BoaAmposen K, Cassady JP. 2012. Nongenetic Factors Affecting Grasscutter production Traits.1. Growth traits. Word App. Sci. J., 18(10): 1412-1424.

Druet VPAD. 2005. Influence de facteurs environnementaux sur la reproduction de la jument. Thèse doc.vet. Lyon, p.52.

Fantodji A, Soro D. 2004. Elevage des Aulacodes: Expérience en Côte d'Ivoire. Guide Pratique. Agridoc. Gret: Paris.
Gayrard V., 2007. Physiologie de la Reproduction des Mammifères. Ecole Nationale Vétérinaire : Toulouse.

Ghavi HZN, Mohit A, Azad N. 2013. Effect of Temperature-Humidity index on productive and reproductive performances of Iranian Holstein Cours. Iran. J. Vet. Res., 14(2): 106-112.

Guerin MV, Wang XJ. 1994. Environnemental temperature has an influence on timing of the first ovulation of seasonal oestrus in the mare. Theriogenology, 42: 1053-1060.

Karamoko Y, Soro S, Soro D, Fantodji A. 2013. Effect of diet on serum Biochemical Parameters and parasitism of Grasscutter (Thryonymys swinderianus) Raised in Côte d'Ivoire. Int. J. P. App. Zoo., 1(3): 255-260

Karamoko Y, Soro S, Traoré B, Fantodji A. 2014. Distributions of Hydrolytic Enzimes activity in the Digestive Tract of Grasscutter. Int. J. P. App. Zoo., 2(1)1-7.

Lebas F. 2011. La Biologie du Lapin. INRA : France.

Poku JPA, Annor SY, Djang-Fordjour KT. 2013. Growth , Reproduction and carcass characteristics of Grasscutters (Thryonomys swinderianus) Fed on Different Levels of Protein Supplement. W. J. Zoo., 8(2): 125-184.

Soro S, Karamoko Y, Soro D, Gonnety TJ, Fantodji A. 2014. Contribution à l'alimentation des jeunes aulacodes (Thryonomys swinderianus) après le sevrage. Les Tech. Lab., 8: 34. 